\title{
Increase in the incidence of dapsone hypersensitivity syndrome - an appraisal
}

\author{
P. NARASIMHA RAO \& T. S. S. LAKSHMI \\ Department of Dermatology, Osmania Medical College/Hospital \\ Hyderabad. India
}

Accepted for publication 15 October 2000

\begin{abstract}
Summary There has been an increase in the reports of dapsone hypersensitivity syndrome (DHS) in the past few years, coinciding with the introduction of multidrug therapy (MDT) for leprosy world-wide. The exact cause of this phenomenon is not clear. We report four cases of DHS observed among 252 leprosy patients on MDT and one case of DHS in a patient taking dapsone for nodulocystic acne in the Dermatology Department of the Osmania General Hospital, Hyderabad, India, between June 1997 and January 1999 with few unusual features. In two of these five patients maculopapular rash was severe and progressed to erythroderma.

Introduction of MDT in 1982 has not only decreased the prevalence of leprosy but also brought about a positive change in the attitude of people which increased the voluntary reporting of leprosy patients. This, coupled with improvements in organization of leprosy control and awareness among medical personnel of DHS, are probably the most important reasons for the increased reporting of DHS in recent years.
\end{abstract}

\section{Introduction}

The terms hypersensitivity and drug allergy describe the allergic reaction that results from sensitization to a particular chemical or a structurally similar one. ${ }^{1}$ Dapsone hypersensitivity syndrome (DHS) is such a reaction, with the median latency, i.e. the time interval from the commencement of therapy to manifestation of the hypersensitivity reaction, of about 4-7 weeks. The manifestations of DHS include some or all of the following: fever, eosinophilia, mononucleosis, lymphadenopathy, hepatitis and exanthematous skin eruption which may progress to a dermatological emergency and rarely to death. ${ }^{2,10}$

In the early years after introduction of dapsone to clinical use, this reaction was reported frequently. However, hypersensitivity reactions to dapsone virtually disappeared from the literature between 1956 and $1980 .^{3}$ After 1980, the number of case reports of DHS increased, coinciding with the introduction of multidrug therapy (MDT) for leprosy. Dapsone, apart from being a first line drug in the treatment of leprosy, is also used in the treatment of many 
other dermatological disorders such as vesiculobullous dermatoses, cutaneous vasculitis, polyarteritis nodosa, nodulocystic acne and cutaneous mycetoma. In all these conditions, dapsone is used at doses of $100 \mathrm{mg} /$ day or more and DHS has also been reported in these patients. $^{3,10}$

Here we report five cases of DHS with few unusual features which were observed in our department during a period of 18 months, and discuss the possible causes of increase in its incidence.

In the Dermatology Outpatient Department of the Osmania General Hospital, Hyderabad, India, a total of 252 leprosy patients were enrolled between June 1997 and January 1999 (165 males and 87 females). All patients were put on WHO recommended MDT. Among these, four patients developed DHS necessitating hospitalization and discontinuation of dapsone. DHS also occurred during the same period in a patient with nodulocystic acne who was treated with dapsone $100 \mathrm{mg} /$ day. None of the patients who were diagnosed as DHS had any other systemic illness. All the leprosy patients who developed DHS were only on MDT and the only systemic medication that the patient with nodulocystic acne was taking was dapsone.

\section{Observations}

In all five patients (Table 1), DHS was observed during the first 5 weeks of treatment and the dose of dapsone was $100 \mathrm{mg} /$ day. Two of the leprosy patients were on multibacillary MDT, whereas the other two were on paucibacillary MDT. The average duration of dapsone therapy before the appearance of DHS was 16 days. Features of DHS were observed earliest in patient 5 , within 2 days, who gave a past history of skin rashes with syncope after taking PB MDT for 2 months at another leprosy clinic before the therapy was discontinued.

All five patients had fever, maculopapular rash associated with itching and generalized lymphadenopathy. Jaundice and hepatomegaly were not present in patient 5 , who had taken dapsone only for 2 days, whereas these symptoms were observed in all the other four patients who had taken dapsone for more than 2 weeks. In two of the five patients, maculopapular rash was so extensive and severe as to have progressed to a state of 'erythroderma'. In patient 3 , tender hepatomegaly was associated with deep jaundice and pulmonary involvement. The signs and symptoms of DHS were mildest in case 5, who had only been treated with dapsone for 2 days.

Liver function tests revealed increased serum levels of bilirubin and SGPT in all four patients with florid DHS. Reversal of serum albumin/globulin ratio was also observed in all four patients. Serological tests for hepatitis B and C were negative and glucose-6-phosphate dehydrogenase levels were within normal limits in all five patients.

Biopsies of the inflamed skin taken from the two patients who had progressed to erythroderma revealed a predominantly mononuclear cell collection around adnexa and small blood vessels. None of these patients demonstrated either leucocytosis or eosinophilia.

\section{Treatment}

Patients with DHS were hospitalized and all the drugs withdrawn, including dapsone. Patients 1 and 3 were put on systemic corticosteroids (30 mg prednesolone per day). Patients 2, 4 and 
Table 1. Clinical features

\begin{tabular}{|c|c|c|c|c|c|}
\hline \multirow[b]{2}{*}{ Clinical features } & \multicolumn{5}{|c|}{ Patient no. } \\
\hline & 1 & 2 & 3 & 4 & 5 \\
\hline Age and sex & 50 years $\mathrm{F}$ & 18 years $\mathrm{M}$ & 22 years $\mathbf{M}$ & 25 years $M$ & 35 years $F$ \\
\hline Diagnosis & LL leprosy & BT leprosy & Indeterminate leprosy & Nodulocystic acne & BT leprosy \\
\hline Therapy & MB MDT & PB MDT & PB MDT & Dapsone + topical therapy & MB MDT \\
\hline $\begin{array}{l}\text { Duration of therapy before } \\
\text { appearance of DHS (days) }\end{array}$ & 20 & 33 & 15 & 12 & 2 \\
\hline Fever & + & + & + & + & + \\
\hline Jaundice & + & + & + & + & - \\
\hline Itching & + & + & + & + & + \\
\hline Lymphadenopathy & + & + & + & + & + \\
\hline Maculopapular rash & + & + & + & + & + \\
\hline Hepatomegaly & + & + & + & + & + \\
\hline Splenomegaly & - & - & - & + & - \\
\hline Erythroderma & + & + & - & - & - \\
\hline Respiratory symptoms & - & - & Crepitations + & - & - \\
\hline
\end{tabular}


5 were put on antihistamines, as well as supportive therapy. All the patients recovered completely. In the patients on corticosteroids, cutaneous lesions resolved in 1-2 weeks (average 9 days), whereas in patients on antihistamines, skin lesions subsided in 2-3 weeks (average 16 days). Corticosteroids were tapered over period of 4 weeks.

Biochemical parameters returned to normal in all patients within 6-8 weeks. None of the patients was rechallenged with dapsone. Clofazamine was substituted in the place of dapsone in patients on paucibacillary MDT. In patients on multibacillary MDT, dapsone was stopped and rifampicin and clofazamine were continued.

\section{Discussion}

Due to the increase in the number of case reports, there has been revival of interest in DHS recently. Moreover, dapsone as a drug has become more important world-wide after recognition of its value in the chemoprophylaxis and treatment of toxoplasmosis and Pneumocystis carini pneumonia in HW infected patients. ${ }^{4}$

DHS is considered as a diagnosis when sudden onset of generalized maculopapular skin rash associated with systemic symptoms, jaundice, haematological abnormalities and generalized lymphadenopathy occurs in patients on dapsone during the first few weeks of its intake. The maculopapular rash with its underlying erythema in severe cases can be associated with inflammation, swelling and later desquamation of the skin. DHS is a clinical diagnosis, as there is no confirmatory laboratory test available currently. No other drug used in MDT of leprosy is known commonly to produce such a reaction.

Although classic DHS can present with all the clinical features, incomplete and atypical forms are encountered occasionally. ${ }^{13}$ The commonly associated clinical features of DHS which are exantheni, fever lymphadenopathy and signs of hepatic dysfunction, were also observed in this study. ${ }^{6,10}$ However, haematological changes such as leucocytosis, eosinophilia and atypical lymphocytosis often associated with $\mathrm{DHS}^{11}$ were not observed in the present study.

Erythroderma is the term applied to any inflammatory skin disease that affects more than $90 \%$ of the body surface area. Erythroderma is observed in severe DHS, the incidence ranging from $76.5 \%$ to $22.2 \%$ as reported in various studies. ${ }^{6,11}$ No correlation between the dosage of dapsone and the occurrence and severity of DHS was observed in various studies. ${ }^{3,7}$ In the present study, two out of five patients with DHS progressed to erythroderma, after taking dapsone for longer periods before the appearance of DHS (33 and 20 days) compared to the others in the study group. It is also interesting to note that DHS was mildest in patient 5 , who had discontinued dapsone on the second day of starting therapy due to prior experience of dapsone sensitivity. It was observed that accidental challenge with dapsone often causes mild symptoms of DHS in patients with hypersensitivity to dapsone, which confirms the diagnosis of this syndrome. ${ }^{6}$ These observations indicate that although the severity of DHS is not dose related, it may be affected by the duration of administration of dapsone after sensitization.

DHS is usually confined to the first 6 weeks of treatment with dapsone. However, it can appear as early as $6 \mathrm{~h}$ in previously sensitized individual to as late as after 6 months of therapy. ${ }^{11}$ In the present, study patient 5 , who had past history of dapsone hypersensitivity, developed DHS within 2 days of starting dapsone. It is important to distinguish true early 
onset DHS from phototoxic and photoallergic reactions to dapsone, which can also occur during the first $24-48 \mathrm{~h}$ of taking the drug in sensitized individuals. ${ }^{12}$

The exact incidence of DHS is not known. Lowe, who was first to report DHS in leprosy patients in 1950 from Nigeria, noted the frequency of dapsone syndrome as $2 \%{ }^{5}$ An incidence of $1.3 \%$ was reported in a study from India. ${ }^{6}$ In the present study, the incidence of DHS among leprosy patients is $1.6 \%$. Richardus and Smith $^{7}$ reported an incidence of DHS of $0.3-0.6 \%$ during monotherapy and 3.6\% after introduction of MDT. Such an increase in the incidence of DHS after introduction of MDT was also reported by other workers. DHS was observed in three out of 24 new cases (12.5\%) who had taken MDT between 1986 to 1995 in a province of China, whereas it was encountered in none of the 1357 leprosy patients before introduction of MDT. ${ }^{8}$ Smith $^{3}$ observed a sudden reappearance and increase of reports of DHS between 1980 and 1986 (27 cases), coinciding with the introduction of MDT for leprosy after the relative absence of cases between 1956 and 1980. However, he also noted that in 16 out of 27 cases, patients were on dapsone monotherapy.

Although unequivocal proof is lacking at present, the interaction of dapsone with other MDT drugs, especially rifampicin, as a cause of DHS cannot be ruled out completely. A post mortem histological examination of a DHS patient demonstrated features consistent with drug induced hepatitis, tubulo-interstitial nephritis and myocarditis. ${ }^{9}$ Although these could have been caused by dapsone toxicity, it was thought that a concomitant adverse reaction to refampicin, which is known to be hepatotoxic and nephrotoxic and possibly capable of predisposing to dapsone syndrome, could not be excluded.

In a review of all published cases of dapsone hypersensitivity world-wide from 1949 to 1995, the authors found only 103 reported cases of DHS during this period of 46 years. ${ }^{10}$ Of these, the highest numbers of cases of DHS were reported after 1980, coinciding with the introduction of MDT for leprosy. ${ }^{3,6-8}$ From the Medline scan and Indian Journal of Leprosy we picked out 24 cases of DHS from seven reports published between years 1996 and 1998, in contrast to the total number of 103 patients of DHS reported in world literature during the period 46 years between 1949 and 1995.

This apparent recent increase in DHS case reports depends on multiple factors, some of may include the following:

1. Increased patient compliance and clinic attendance after introduction of $\mathrm{MDT}^{14}$ which brought about better detection of DHS than before.

2. The introduction of MDT has also contributed to the improvements in the organization of leprosy control programmes ${ }^{15}$ and level of job satisfaction in the health personnel, ${ }^{16}$ which could have resulted in the increased reporting of DHS.

3. Awareness of DHS among medical personnel due to increased usage of dapsone in various indications other than leprosy.

It may be of interest to note that all these recent cases of DHS in leprosy patients between 1996 and 1998 were reported from departments of Dermatology, Medicine, Pharmacology or Forensic Medicine of teaching hospitals or institutions and none from leprosy clinics. This probably reflects the increased utilization of services of tertiary medical care centres by leprosy patients, resulting from the increased referral of these patients for specialist care. The recent increase in the reporting of DHS from these centres indicates the increased interest of allied medical specialties in DHS, apart from the fact that these centres are better equipped than a peripheral leprosy clinic to investigate, record and report such cases systematically. 


\section{References}

${ }^{1}$ Klaassen CD. Principles of toxicology. In: Gilman AG, Rall TW, Nies AS et al. (eds) The pharmacological basis of therapeutics, 8th edition. McGraw Hill, NY, 1992, pp 49-61.

2 Jopling WH. Side effects of anti-leprosy drugs in common use. Lepr Rev, 1983; 54: 261-270.

${ }^{3}$ Srnith WCS. Are hypersensitivity reactions to dapsone becoming more frequent? Lepr Rev, 1988; 59: 53-58.

${ }^{4}$ Gold JWM. The diagnosis and management of HIY infection. Med Clin N Am, 1996; 80: 1283-1307

${ }^{5}$ Lowe J. Diaminodiphenyl sulphone in the treatment of leprosy. Lancet, 1951; 1: 1343-1344.

${ }^{6}$ Rege VL Shukia P Mascarenhas W. Dapsone syndrome in Goa. Ind J Lepr, 1994; 66: 59-64.

${ }^{7}$ Richardus JJ Smith TC. Increased incidence in leprosy of hypersensitivity reactions to dapsone after introduction of multidrug therapy. Lepr Rev, 1989; 60: 267-273.

${ }^{8}$ Guanwei G. DDS syndrome when taking NMT. China Lepr J, 1995; 11: 200-201.

${ }^{9}$ Lau G. A fatal case of drug induced multi-organ damage in a patient with Hansen's disease. Dapsone syndrome or rifampicin toxicity? Forens Sci Int, 1995; 73: 109-115.

${ }^{10}$ Wogel G, Gobel K, Blasum C. Hypersensitivity syndrome to dapsone - an epidemiological review. In: Abstracts of ILEP 15th International Leprosy Congress, Beijing, 1998, CH-31.

${ }^{11}$ Kumu RH, Kumar MV, Thapa DM. Dapsone syndrome - a five year retrospective analysis. Ind J Lepr, 1998; 70: 271-276.

12 Joseph MS. Photodermatitis provoked by dapsone. A case report. Lepr Rev, 1987; 58: 425-428.

13 Jaswal R, Thami GP, Kanwar AJ. Dapsone syndrome - an incomplete form. Ind J Lepr, 1998; 70: 229-230.

${ }_{14}^{14}$ Gupte MD. Elimination of leprosy: forecasts and projections. Ind J Lepr, 1994; 66: 19-35.

${ }^{15}$ Noordeen SK. Elimination of leprosy as a public health problem. Ind J Lepr, 1994; 66: 1-10.

${ }^{16}$ Premkumar R, Dave SL. Impact of multidrug therapy on health personnel in their level of job satisfaction. Ind $J$ Lepr, 1993; 65: 429-438. 